\title{
On Decoding and Equalization of Multidimensional Trellis-Coded OFDM Systems
}

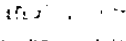 \\ ' Pei-Chun Liu \\ -Graduate Institute of Communication Engineering \\ National Taiwan University \\ angelpei@isantos.ee.ntu.edu.tw \\ 1'Sec.4 Roosevelt Rd. Taipei 106, Taiwan

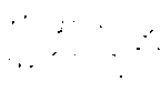

Abstract-In this paper, we develop an effective method,
Multicarrier Parallel Decision Feedback Decoding (MC-PDFD),
to combat the residual ISI (intersymbol interference) effect in
OFDM systems employing multi-dimensional trellis-coded
modulation (TCM) scheme. The proposed method operates on
reduced-state trellises with modified branch metric in order to
accommodate OFDM and other multicarrier communications.
MC-PDFD combines the task of trellis decoding and
equalization altogether and removes the residual ISI
simultaneously with decoding process. Simulation results show
that MC-PDFD works well in different channel conditions with
perfect channel information and degrades slightly with
non-perfect channel-information.

Index Terms - Cömbine, Decoding, Equalization, Intersymbol Interference 'Channel, Multidimension, MLSE, OFDM, PDFD, RSSE, Set Partitioning Principle, Trellis-Coded Modulation, TCM.

\section{[i: $: .$. I. INTRODUCTION}

The rapid growth of communication for video, voice, data, and anlso the equally rapid pervasion of mobile telephoney justify great expectations for mobile multimedia service, which requires the transmission of very high data rates over broadband radio channels. OFDM has been considered a good modulation candidate for such radio channels owing to its robustness against frequency selective fading. It turns the original channel into a number of flat fading narrow-band subchannels and simplifies the equalizer structure. However, the problem of residual ISI (intersymbol interference) caused by multipath effects with long channel memory still exists in OFDM systems and thus developing methods rather than simple equalization is necessary.

On the other hand, trellis-coded modulation (TCM) is always receiving great interests and its behavior in the presence of ISI also draws lots of attention [1][2]. To solve both problems of trellis decoding and ISI in single carrier cases, RSSE (reduced-state sequence estimation[2]) has been proposed, which employed set partitioning principle to merge states in trellis diagram that combines states from both channel and trellis code states. In this way, RSSE avoided complexity from MLSE.

\author{
Kwang-Cheng Chen \\ Department of Electrical Engineering \\ National Taiwan University \\ chenkcocc.ee.ntu.edu.tw \\ 1 Sec.4 Roosevelt Rd. Taipei 106, Taiwan
}

However, when it comes to multicarrier cases, such as OFDM systems, with multi-dimensional trellis code, some modification should be made both in how to merge states and how to compute branch metric in order to extend the idea of RSSE and take care of trellis decoding and the problem of residual ISI simultaneously.

In this paper, based on the idea of RSSE and multicarrier nature of OFDM, we develop a method called MC-PDFD (Multicarrier Parallel Decision Feedback Decoder) that deals with both residual ISI and multi-dimensional trellis decoding in OFDM systems altogether. The paper is arranged as follows: Section II presents our system model; section III illustrates details of MC-PDFD, including principles, procedures and examples; section IV gives the simulation results; and finally a short conclusion is drawn in section $\mathrm{V}$.

\section{SYSTEM MODEL}

Figure 1 is our OFDM system model. Incoming serial data is first serial-to-parallel converted. The resulting parallel data is then encoded by a 4-dimensional trellis coded modulation (4D-TCM) encoder and mapped with a QAM mapper. After mapping, $\mathrm{N}$-point inverse fast Fourier transform (IFFT) operation interleaves these mapped points onto different subcarriers and the last $G$ samples are put ahead as the Guard interval. At the end of the transmitter, parallel-to-serial converter converts IFFT output back to serial, and send it to the channel. Here we use a multipath channel with memory length $K, K>G$

In the receiver, the inverse operations of the transmitter are performed, including serial-to-parallel conversion, guard interval removal, and fast Fourier transform. Here we assume that synchronization block works perfectly and the channel information is available so that we can concentrate on dealing with the problem of residual ISI. After passing through the FFT block, these parallel results are serially fed into the MC-PDFD (Multicarrier Parallel Decision Feedback Decoder), where the tasks of removing residual ISI and trellis decoding are performed at the same time. Output points from PDFD can then be converted back to bit stream according to one-to-one functions in the 4-D TCM encoder and this bit stream is used for final bit error rate calculation. 


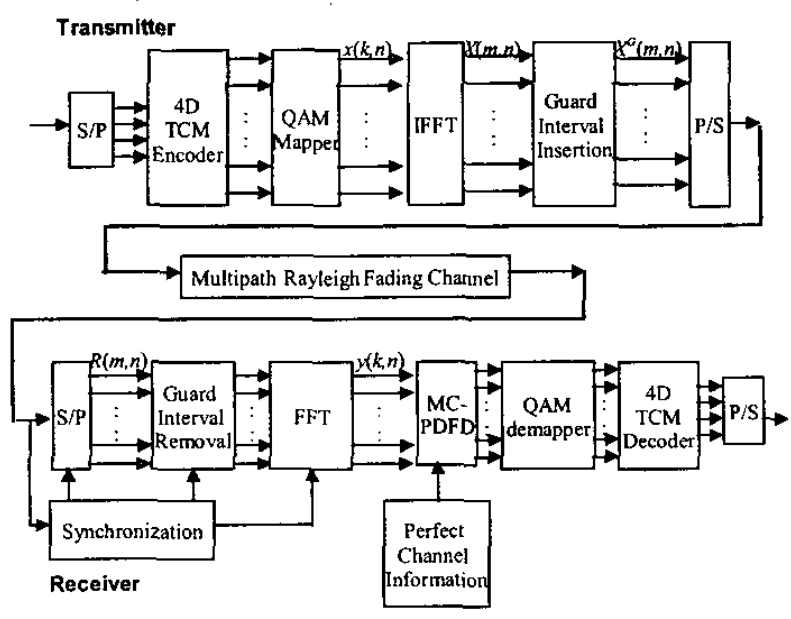

Fig. 1. System Model.

\section{MC-PDFD}

The goal of MC-PDFD is to remove residual ISI while performing trellis decoding in multicarrier communication systems. Based on the idea in RSSE [2], we develop decoding trellis and branch metric suitable for TCM-OFDM systems. In this section, we first construct trellises for MC-PDFD, then we derive branch metric that combines residual ISI removal and trellis decoding together. In the latter part of this section, the procedures of MC-PDFD according to the branch metric in the former part is presented and a simple example is given before closing this section.

\section{A. Construct MC-PDFD trellises}

In [2], we have known that the complexity of ML super trellis which combines both code states and channel states can be reduced if channel states are merged according to set partitioning principle.

In MC-PDFD, channel states in each code dimension are merged so that the resulting trellis is reduced to encoder trellis with parallel transitions on each branch. Figure 2 illustrates the reduced-trellis process from ML super trellis to MC-PDFD trellis. The parameters we use are as follows:

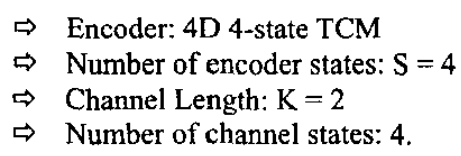

The ML super trellis in this case has a total of $4 \times 4=16$ states, as shown in Figure 2(a). With class A set partitioning and channel states merging, ML super trellis can be reduced to 8 -state trellis in Figure 2(b). When channel states all merge together, we obtain the trellis of MC-PDFD as shown in
Figure 2(c), which is exactly the encoder trellis. All merged states will be taken into account in the branch metric in order to remove residual ISI.

\section{B. Derive branch metric}

To achieve ISI suppression based on a trellis coded system, and also taken into account that the case now is a multi-carrier system, necessary modification should be made in the branch metric.

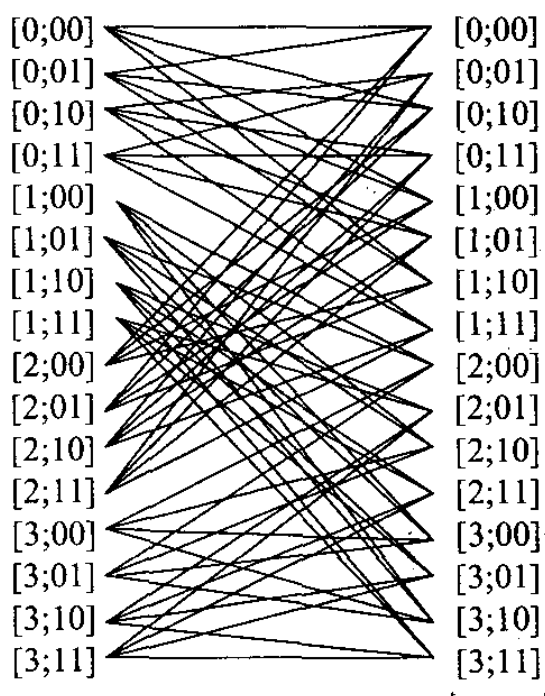

$\cdot$

$+$

i, , ,

21

$+$

Fig. 2 (a).16-state ML super trellis

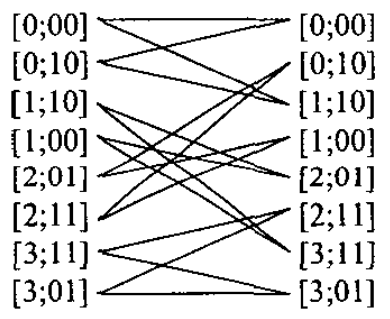

(b) 8-state reduced trellis

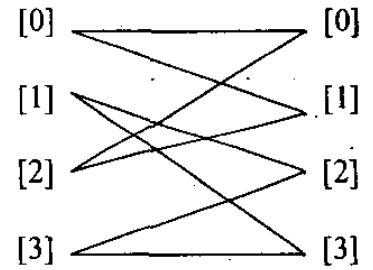

(c) 4-state MD-PDFD trellis
According to figure 1., outputs after guard interval insertion at the transmitter side can be expressed as

$$
X^{G}(m, n)=\left\{\begin{array}{l}
X(m+N-G, n), 0 \leq m \leq G-1 \\
X(m-G, n), G \leq m \leq N+G-1
\end{array}\right.
$$

where

$$
X(m, n)=\frac{1}{N} \sum_{k=0}^{N-1} x(k, n) \exp (j 2 \pi k m / N),(3.2)
$$

After multipath fading channel, we have 
$Y^{G}(m, n)=\sum_{l=0}^{N+G-1} X^{G}(l, n) h(m-l)$.

$m=0,1, \ldots, N+G+K-1$

where $h()$ is channel impulse response.

Now it is trivial that the last $K$ terms of $Y^{G}(m, n)$ would interfere with next block after guard interval removal, i.e. the residual ISI teerm is

$$
\begin{aligned}
\text { ISI }_{t, n+1}= & Y^{G}(m, n) \\
= & \sum_{l=0}^{N+G-1} X^{G}(l . n) h(m-l) \\
= & \sum_{l=0}^{G-1} X(l+N-G, n) h(m-l) \\
& +\sum_{l=G}^{N+G-1} X(l-G, n) h(m-l) \\
& N+G \leq m \leq N+G+K-1 .
\end{aligned}
$$

The subscripts $t$ and $n+1$ represent the ISI before FFT operation in the OFDM block $n+1$.

Note that in equation (3.4), $m$ ranges from $N+G$ to $N+G+K-1$. Within this range, $Y^{G}(m, n)$ is related to $Y(m, n)$ according to

$$
Y(m, n)=Y^{G}(m+G, n) .
$$

$\mathrm{ISI}_{t, n+1}$ can thus be changed to the following form, with modified boundary.

$$
\begin{aligned}
\operatorname{ISI}_{t, n+1} & =Y(m, n) \\
& =Y^{G}(m+G, n) \\
& =\sum_{i=0}^{G-4} X(l+N-G, n) h(m+G-l) \\
& +\sum_{l=G}^{N+G-1} X(l-G, n) h(m+G-l) \\
& =\sum_{i=-G}^{-1} X(N+l, n) h(m-l)+\sum_{i=0}^{N-1} X(l, n) h(m-l) \\
& N \leq m \leq N+K-1 .
\end{aligned}
$$

Finally the residual ISI term after FFT can be obtained as

$$
\begin{aligned}
\text { ISI }_{k, n+1} & =\sum_{m=N}^{2 N-1} Y(m, n) \exp (-j 2 \pi k m / N) \\
& =\sum_{m=N}^{N+K-1} Y(m, n) \exp (-j 2 \pi k m / N) \\
& =\sum_{m=N+G}^{N+K-1}\left[\sum_{l=-G}^{-1} X(N+l, n) h(m-l)\right. \\
& \left.+\sum_{l=0}^{N-1} X(l, n) h(m-l)\right] \exp (-j 2 \pi k m / N)
\end{aligned}
$$

$$
\begin{aligned}
& =\sum_{m=N+G}^{N+K-1} \sum_{l=N-K+G}^{N-1} X(l, n) h(m-l) e^{-j \frac{2 \pi k m}{N}} \\
& =\sum_{l=1}^{K-G} X(N-l, n) \sum_{m=1}^{K-1} h(K-m+1) e^{-j \frac{2 \pi k}{N}(K-l-m+1)},
\end{aligned}
$$

where the fourth equality holds because $h(m-l)$ is nonzero only for $m-l \leq K$

Consequently, ISI term in equation (3.6) is the unwanted part in the received signals and should be subtracted. With modification for OFDM systems and subtraction of residual ISI term, the branch metric of MC-PDFD becomes

$$
\begin{aligned}
& b(k ; n)=1 y(k, n)-x(k, n) H_{k}- \\
& \left.\sum_{l=1}^{K-G} X(N-l, n-1) e^{-j \frac{2 n k}{N}(K-l)} \sum_{m=1}^{K-l} h(K-m+1) e^{j \frac{2 \pi k m}{N}}\right|^{2},
\end{aligned}
$$

where $H($.$) is channel information in frequency domain..$

\section{Procedures of MC-PDFD}

Given the branch metric above, the decoding procedure of PDFD for OFDM systems is as follows.

\section{1) Calculate $X(m, n-1)$}

For a certain trellis state with a newly received $y(k, n)$, our first task is to calculate the ISI term corresponding to this state at this stage. From equation (3.7), one of the ISI components is $X(m, n-1)$, which is the Fourier transform of signal block $n-1$ :

$$
X(m, n-1)=\sum_{k=0}^{N-1} x(\dot{k}, n-1) e^{-j \frac{2.2 k m}{N}}
$$

Note that in (3.8), $x(k, n-1), k=0 \sim N-1$, are the decision points that we obtained in the last OFDM biock and stored in each path history. Therefore, we need to trace back to the correct index indicating block $n-1$ along the surviving path of the current state and draw out those necessary $x(k, n-1)$ to calculate $X(m, n-1)$. Since path histories differ form each other, the resulting $X(m, n-1)$ are different. Basically, each time we start decoding a new OFDM block, a new $X(m, n-1)$ table is updated with new $X(m, n-1)$ value at each state. When the trace-back index stops, the corresponding $X(m, n-1)$ is known by looking up this table.

\section{2) Calculate the ISI term}

As we can see in equation (3.7), the complete ISI term includes channel information and some necessary phase modifications. The second step in decoding is to gather all the information and calculate the ISI value for the received $y(k, n)$. In fact, here another table of size $N x(K-G)$ is built to store 
results of $\sum_{l=1}^{K-G K-l} \sum_{m=1} h(K-m+1) e^{j \frac{2 \pi k m}{N}}$. This table can speed up ISI calculation by removing complex multiplications in our decoding process.

\section{3) Select the closest point from parallel transitions.}

Recall that there are usually uncoded bits in a TCM encoder and thus parallel transitions exist in our decoding trellis. We calculate branch metric values of these parallel transitions using the same ISI value but different $x(k, n)$ corresponding to each transition. The one with smallest branch metric value is the surviving transition and is used to compare with other state inputs

4) Continue as usual Viterbi algorithm.

The remaining work is the same as conventional Viterbi algorithm, including path metric accumulation, path metric comparison, surviving path selection, and trellis update. The final decision would be the one with the smallest accumulated metric value, and points in its memory are used to mapped back to bit stream.

Note that in the case of multi-dimensional TCM decoding, all above stay unchanged except that the branch metric of multi-dimensional TCM is the sum of its constituent 2D branch metric. For example, the branch metric of 4D-TCM is composed of two elements, one being calculated from the first received $2 \mathrm{D}$ point, say, $\mathrm{y}(\mathrm{k}, \mathrm{n})$, and the other from the second received $2 D$ point, $y(k+1, n)$.

\section{Example of MC-PDFD}

Here we use a simple example to show how MC-PDFD functions. The notations are the same as in Figure 1, and the parameters are:

$$
\begin{aligned}
& \Rightarrow \text { Channel Length: } \quad K=3 \\
& \Rightarrow \text { Guard Interval: } G=1 \\
& \Rightarrow \text { FFT point: } N=64 .
\end{aligned}
$$

After removing the guard interval at the receiver front end and having these data fast Fourier transformed, we obtain the FFT outputs as

$$
y(k, n)=\sum_{m=1}^{64} R(m, n) \exp (-j 2 \pi k m / 64), k=0,1, \ldots, 63 .
$$

For $k=0$,

$$
\begin{aligned}
y(0, n)= & x(0, n) H_{0}+ \\
& \left\{X(63, n-1) \sum_{l=2}^{3} h(l)+X(62, n-1) h(3)\right\}+\text { ICI terms. }
\end{aligned}
$$

The terms in the bracket are residual ISI which should be subtracted when calculating branch metric. For $k=1$,

$$
\begin{aligned}
& y(1, n)=x(1, n) H_{1} e^{-j \frac{2 \pi}{N}} \\
& +\left\{\left[X(63, n-1)\left[h(2)+h(3) e^{-j \frac{2 \pi}{N}}\right]+X(62, n-1) h(3)\right]\right\} e^{-j \frac{2 \pi}{N}}
\end{aligned}
$$

+ ICI terms.

Those in the square bracket represent residual ISI. Compared to the result in subcarrier 0 , we see that terms appeared in residual ISI are both $X(63, n-1)$ and $X(62, n-1)$ with channel parameters $h(2)$ and $h(3)$. In fact, the situation remains the same if we go on with subcarrier index $k=2,3, \ldots, 63$. This is because guard interval length is shorter than the channel length by two, and thus the last two samples of the previous OFDM block interfere with the current OFDM block. Furthermore, the number of related channel parameters with $X(m, n-1)$ in residual ISI is the difference between channel length $K$ and the position of $X(m, n-1)$ counting backwards, a result of convolution operation of transmitted sequence and channel in time domain. These are exactly in accordance with the general form derived in (3.6). The branch metric in this case is thus

$$
\begin{aligned}
& b(k ; n)=\mid y(k, n)-x(k, n) H_{k} \\
& -\left.\left[X(63, n-1)\left(h(2)+h(3) e^{-j \cdot \frac{2 n k}{N}}\right)+X(62, n-1) h(3)\right]\right|^{2}, \\
& \mathrm{k}=0,1,2, \ldots, 63 .
\end{aligned}
$$

Therefore, we first need to calculate $X(62,$.$) and X(63,$. using $x_{k}$ 's stored in the path history and then combine channel information $h(),. H($.$) to compute branch metric for every$ transition corresponding to the received signal $y(k, n)$.The rest is just normal Viterbi Algorithm which searches for survival path and outputs its history as the decoding results.

\section{SimUlation RESUlts}

Simulation is done with several channel cases and system parameters. As references, the performances of one-tap decision feedback equalizer (DFE) optimal in the sense of minimum mean square error (MMSE) are also plotted.

Figure 3 illustrates the performance of MC-PDFD with 4 subcarriers (guard interval length $=1$ ) and 4-D TCM under a fading channel with memory length equal to 2 .

Another case is shown in Figure 4 . with number of subcarriers $N=64$, guard interval length $G=4$, and channel memory $\mathrm{K}=4$. Simulation is also done with 4-D TCM OFDM systems.

From the simulation results, we can see that MC-PDFD outperforms MMSE-DFE optimal in the sense of minimizing mean square error and removes residual ISI effectively in the process of trellis decoding. Note again that MC-PDFD completes its work together with trellis decoding and therefore, in our OFDM system, no other additional block is necessary to handle problems about residual ISI. 


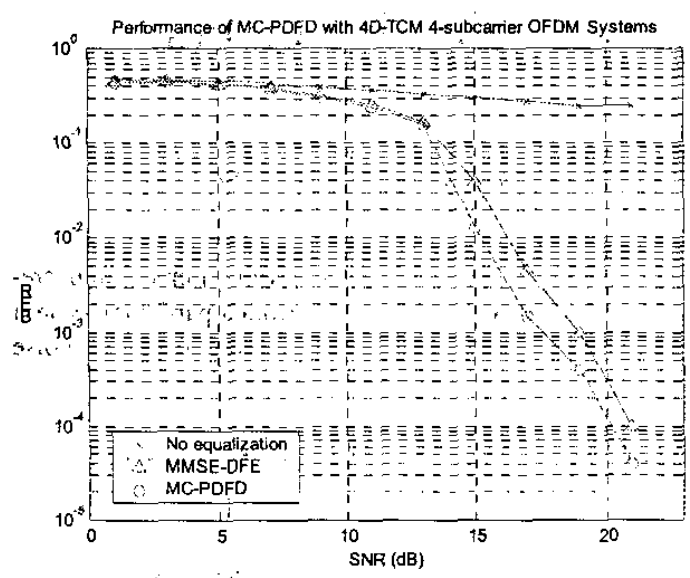

Fig. 3. Performance of $M C$ - $P D F D .(N=4, G=1, K=2)$

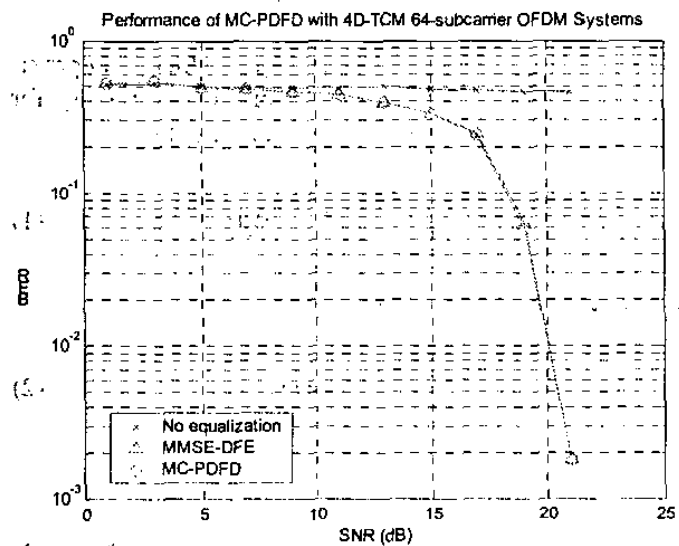

Fig. 4. Performance of MC-PDFD. $(\mathrm{N}=64, \mathrm{G}=2, \mathrm{~K}=4)$

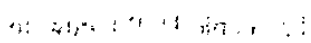

$\therefore=\therefore \cdot \cdots \cdot \mathrm{V}$. CONCLUSION

MC-PDFD is proposed to solve the problem of residual ISI in trellis-coded OFDM communication systems when channel length is longer than the guard interval. It utilizes different feedbacks from the decoding trellis history and thus avoids the problem of error propagation in conventional decision feedback equalizers. Simulation results also show that MC-PDFD has good performances and thus can be considered a good candidate of equalization/decoding in trellis-coded OFDM system ${ }^{3}$

$$
\begin{aligned}
& \cdots \\
& \therefore
\end{aligned}
$$

\section{REFERENCES}

[1] P. R. Chevillat, and E.Eleftheriou, "Decoding of Trellis-Encoded Signals in the Presence of Intersymbol Interference and Noise," IEEE Trans. Comm., Vol. COM-37. pp.669-676, July, 1989.

[2] M. V. Eyuboḡlu, S. U. H. Qureshi, "Reduced-State Sequence Estimation with Set Partitioning and Decision Feedback," IEEE Trans. Comm., Vol. COM-36, pp.13-19, Jan. 1988

[3] L. F. Wei, "Trellis-Coded Modulation with Multidimensional
Constellations," IEEE Trans. Inform. Theory, Vol. IT-33, pp.483- 501, July, 1987.

[4] H. Rohling T May, K. Brininghaus, and R. Grünheid, "Broad-band OFDM radio transmission for multimedia applications," Proceedings of the IEEE, Vol. 87, No. 10, pp.1778-1788, Oct. 1999.

[5] M. V. Eyuboğlu, S. U. H. Qureshi, "Reduced-State Sequence Estimation for coded modulation on intersymbol interference channels," IEEE JSAC., Vol. SAC-7, No. 6, pp.989-995, Aug. 1989.

[6] M. V. Eyuboğlu, S. U. H. Qureshi, "Reduced-state sequence estimation for trellis-coded modulation on intersymbol interference channels," Proc. GLOBECOM'88, Hollywood, FL, Nov.1988.

[7] R. van Nee and R. Prasad, OFDM for wireless multimedia communications, Artech House Publishers, 2000

[8] J. G. Proakis, Digital Communications, $3^{\text {rd }}$ edition, MC-Graw Hill, 1995. 